Nature Reviews Cancer | AOP, published online 9 August 2007; doi:10.1038/nrc2213

$\Rightarrow$ BREAST CANCER

\title{
A striking resemblance
}

DOI:

10.1038/nrc2213
How similar can a mouse be to a human? Jos Jonkers and colleagues have created mice with somatic loss of $\underline{B R C A 1}$ and $p 53$ that develop tumours that look much like human basal-like breast cancer that arises from $B R C A 1$ mutation carriers. Piet Borst and colleagues have validated the use of this mouse model by using it to further our understanding of chemotherapy resistance mechanisms.

Breast cancers that arise in women who carry mutations in BRCA1 frequently have mutations in TP53 and have a basal-like phenotype. Various mouse strains with mutations in Brcal have been created, and some have been combined with $\operatorname{Trp} 53$ mutations, but none of these mice develop breast cancer that resembles BRCA1-mutated basal-like human cancers. To get around the fact that conventional Trp53 knockout mice develop lymphomas and sarcomas rather than epithelial tumours, Jonkers and colleagues used K14cre mice, which express the Cre recombinase only in epithelial tissues, to develop mice with epithelial-specific loss of Trp53 and/or Brca1. Mice lacking both p53 and BRCA1 (K14cre;Brca1 ${ }^{F / F} ; \operatorname{Trp} 53^{F / F}$ mice) developed mammary tumours more quickly than those lacking p53 only (K14cre; $\operatorname{Trp} 53^{F / F}$ mice), and K14cre;Brca1 ${ }^{F / F}$;rpp53 $3^{F / F}$ mice had reduced survival compared with mice retaining one wild-type allele of either gene. Histopathologically, about $90 \%$ of tumours from $\mathrm{K} 14$ cre;Brca1 ${ }^{F / F} ; \operatorname{Trp} 53^{F / F}$ mice resembled human breast tumours with hereditary BRCA 1 mutations. Unsupervised clustering of gene-expression profiles of 32 K14cre;Brca1 ${ }^{F / F} ; \operatorname{Trp} 53^{F / F}$ tumours,
$21 \mathrm{~K} 14 c r e ; \operatorname{Trp} 53^{F / F}$ tumours, 16 oestrogen receptor (ER)-negative $B R C A 1$-mutated human tumours and 28 ER-negative sporadic human tumours revealed that the tumours from K14cre;Brca1 ${ }^{F / F}$; $\operatorname{Tr} p 53^{F / F}$ mice co-cluster with BRCA1-mutated human tumours, and those from K14cre; $\operatorname{Trp} 53^{F / F}$ mice co-cluster with the ER-negative sporadic human tumours.

Borst and colleagues have used the $\mathrm{K} 14 \mathrm{cre} ; \mathrm{Brcal}^{\mathrm{F} / F}$; $\operatorname{Trp} 53^{\mathrm{F} / \mathrm{F}}$ mice to examine in vivo responses to chemotherapy that is frequently used to treat breast cancer. Mice treated with either the anthracycline doxorubicin or the taxane docetaxel at the maximum tolerated dose (MTD) had heterogenous responses, but all eventually developed resistance. Furthermore, orthotopically transplanted pieces of tumour in wild-type mice resembled the original tumours and had the same responses to chemotherapy, which could make the model more useful for tumour intervention studies. Examination of mechanisms of doxorubicin resistance revealed overexpression of Mdr1a, a homologue of $M D R 1$, which encodes P-glycoprotein, and increased efflux of a labelled MDR1A substrate was seen in vivo. These tumours were also cross-resistant to docetaxel, which was expected as docetaxel is also a P-glycoprotein substrate. The authors also investigated the response of $\mathrm{K} 14 \mathrm{cre}$; $\mathrm{Brca1}^{F / F}$; $\operatorname{Tr} p 53^{F / F}$ mice to cisplatin treatment.

Although the mice did not develop resistance, tumours could not be eradicated with cisplatin. They propose that the remaining cells could be tumour-initiating cells with stem cell-like properties.

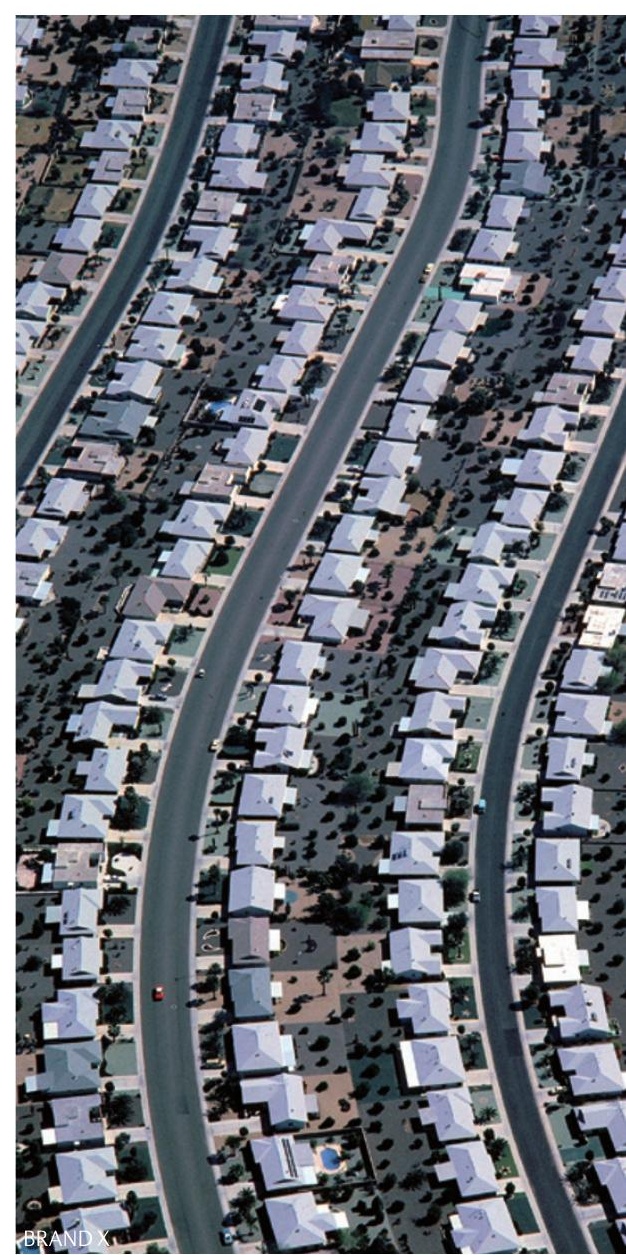

The tumours in these mice bear a striking resemblance to human tumours, and because they also acquire chemotherapy resistance much like human tumours, these mice will hopefully be a useful tool for better understanding the biology and therapy of BRCA1-mutated basal-like human breast cancers. Sarah Seton-Rogers

ORIGINAL RESEARCH PAPERS Liu, X. et al. Somatic loss of BRCA1 and $p 53$ in mice induces mammary tumours with features of human BRCA1-mutated basal-like breast cancer. Proc. Natl Acad. Sci. USA 104, 12111-12116 (2007)| Rottenberg, S. et al. Selective induction of chemotherapy resistance of mammary tumours in a conditional mouse model for hereditary breast cancer. Proc. Natl Acad. Sci. USA 104, 12117-12122 (2007) 